\title{
Strong laws for weighted sums of random variables satisfying generalized Rosenthal type inequalities
}

\author{
Yanchun $\mathrm{Yi}^{1}$, Pingyan Chen ${ }^{2}$ and Soo Hak Sung ${ }^{3^{*}}$ (D)
}

${ }^{\text {*Correspondence: }}$ sungsh@pcu.ac.kr

${ }^{3}$ Department of Applied

Mathematics, Pai Chai University, Daejeon, South Korea

Full list of author information is available at the end of the article

\begin{abstract}
Let $1 \leq p<2$ and $0<\alpha, \beta<\infty$ with $1 / p=1 / \alpha+1 / \beta$. Let $\left\{X_{n}, n \geq 1\right\}$ be a sequence of random variables satisfying a generalized Rosenthal type inequality and stochastically dominated by a random variable $X$ with $E|X|^{\beta}<\infty$. Let $\left\{a_{n k}, 1 \leq k \leq n, n \geq 1\right\}$ be an array of constants satisfying $\sum_{k=1}^{n}\left|a_{n k}\right|^{\alpha}=O(n)$. Marcinkiewicz-Zygmund type strong laws for weighted sums of the random variables are established. Our results generalize or improve the corresponding ones of Wu (J. Inequal. Appl. 2010:383805, 2010), Huang et al. (J. Math. Inequal. 8:465-473, 2014), and Wu et al. (Test 27:379-406, 2018).
\end{abstract}

MSC: $60 \mathrm{~F} 15$

Keywords: Strong law of large numbers; Weighted sum; Widely orthant dependent random variable

\section{Introduction}

Let $\left\{X_{n}, n \geq 1\right\}$ be a sequence of random variables, and let $\left\{a_{n k}, 1 \leq k \leq n, n \geq 1\right\}$ be an array of constants. The limiting behavior of weighted sums $\sum_{k=1}^{n} a_{n k} X_{k}$ is useful in statistics since many linear statistics such as least-squares estimators and nonparametric regression function estimators are of the form of the weighted sums.

The classical Marcinkiewicz-Zygmund strong law of large numbers states that if $\left\{X_{n}, n \geq 1\right\}$ is a sequence of independent and identically distributed (i.i.d.) random variables with $E X_{1}=0$ and $E\left|X_{1}\right|^{p}<\infty$ for some $1 \leq p<2$, then $n^{-1 / p} \sum_{k=1}^{n} X_{k} \rightarrow 0$ a.s. Cuzick [5] and Bai and Cheng [2] ( $p=1$ and $1<p<2$, respectively) obtained a MarcinkiewiczZygmund type strong law of large numbers for weighted sums of i.i.d. random variables, i.e., they proved that

$$
n^{-1 / p} \sum_{k=1}^{n} a_{n k} X_{k} \rightarrow 0 \quad \text { a.s. }
$$

(c) The Author(s) 2020. This article is licensed under a Creative Commons Attribution 4.0 International License, which permits use, sharing, adaptation, distribution and reproduction in any medium or format, as long as you give appropriate credit to the original author(s) and the source, provide a link to the Creative Commons licence, and indicate if changes were made. The images or other third party material in this article are included in the article's Creative Commons licence, unless indicated otherwise in a credit line to the material. If material is not included in the article's Creative Commons licence and your intended use is not permitted by statutory regulation or exceeds the permitted use, you will need to obtain permission directly from the copyright holder. To view a copy of this licence, visit http://creativecommons.org/licenses/by/4.0/. 
when $\left\{X_{n}, n \geq 1\right\}$ is a sequence of i.i.d. random variables with $E X_{1}=0$ and $E\left|X_{1}\right|^{\beta}<\infty$, and $\left\{a_{n k}, 1 \leq k \leq n, n \geq 1\right\}$ is an array of constants satisfying

$$
\sum_{k=1}^{n}\left|a_{n k}\right|^{\alpha}=O(n)
$$

where $\alpha, \beta>0$ and $1 / p=1 / \alpha+1 / \beta$. Note that if we set $a_{n k}=1$ for all $1 \leq k \leq n$ and $n \geq 1$, then (1.1) reduces to the Marcinkiewicz-Zygmund strong law of large numbers. However, the moment condition is strengthened to $E\left|X_{1}\right|^{\beta}<\infty$.

The Cuzick-Bai-Cheng result has been generalized and extended in several directions. Chen and Gan [3] generalized it by considering the norming sequence as $\left\{n^{1 / p} l(n)\right\}$, where $l(x)>0$ is a slowly varying function. Wu [12] extended it to negatively orthant dependent random variables $\left\{X_{n}, n \geq 1\right\}$ which are stochastically dominated by a random variable $X$ satisfying $E|X|^{\beta}<\infty$, i.e.,

$$
P\left(\left|X_{n}\right|>x\right) \leq D P(|X|>x) \text { for all } n \geq 1 \text { and } x>0
$$

where $D>0$ is a constant. Huang et al. [6] extended it to $\varphi$-mixing random variables under a mixing rate condition $\sum_{n=1}^{\infty} \varphi^{1 / 2}(n)<\infty$. Recently, Wu et al. [13] extended it to random variables satisfying a Rosenthal type inequality

$$
\begin{aligned}
& E\left|\sum_{k=1}^{n}\left(f_{n k}\left(X_{k}\right)-E f_{n k}\left(X_{k}\right)\right)\right|^{s} \\
& \quad \leq C_{s}\left\{\sum_{k=1}^{n} E\left|f_{n k}\left(X_{k}\right)\right|^{s}+\left(\sum_{k=1}^{n} E\left(f_{n k}\left(X_{k}\right)\right)^{2}\right)^{s / 2}\right\}, \quad \forall n \geq 1, s \geq 2,
\end{aligned}
$$

where $C_{s}$ is a positive constant depending only on $s$, and $\left\{f_{n k}(x), 1 \leq k \leq n, n \geq 1\right\}$ is an array of nondecreasing functions. However, the moment condition of Wu et al. [13] is strengthened to $E|X|^{u}<\infty$ for some $u>\beta$. In this paper, we improve the result of Wu et al. [13] by weakening the moment condition as $E|X|^{\beta}<\infty$.

The following condition is a Rosenthal type inequality for the maximums of partial sums, which is stronger than (1.3).

$$
\begin{aligned}
E \max _{1 \leq m \leq n}\left|\sum_{k=1}^{m}\left(f_{n k}\left(X_{k}\right)-E f_{n k}\left(X_{k}\right)\right)\right|^{s} \\
\quad \leq C_{s}\left\{\sum_{k=1}^{n} E\left|f_{n k}\left(X_{k}\right)\right|^{s}+\left(\sum_{k=1}^{n} E\left(f_{n k}\left(X_{k}\right)\right)^{2}\right)^{s / 2}\right\}, \quad \forall n \geq 1, s \geq 2 .
\end{aligned}
$$

When $\left\{X_{n}, n \geq 1\right\}$ are independent random variables, (1.3) holds by the Rosenthal [7] inequality, and (1.4) also holds by combining the Rosenthal [7] inequality with the Doob inequality. It is also well known that (1.3) or (1.4) holds for some classes of dependent random variables. If $\left\{X_{n}, n \geq 1\right\}$ are negatively orthant dependent random variables, then (1.3) holds (see Asadian et al. [1]). If $\left\{X_{n}, n \geq 1\right\}$ are negatively associated or $\rho^{*}$-mixing random variables, then (1.4) holds (see Shao [8] and Utev and Peligrad [9], respectively) and hence 
(1.3) holds. When $\left\{X_{n}, n \geq 1\right\}$ are $\varphi$-mixing random variables with $\sum_{n=1}^{\infty} \varphi^{1 / 2}(n)<\infty$, (1.4) holds (see Wang et al. [11]).

Clearly, the following two inequalities are more general than (1.3) and (1.4), respectively:

$$
\begin{aligned}
& E\left|\sum_{k=1}^{n}\left(f_{n k}\left(X_{k}\right)-E f_{n k}\left(X_{k}\right)\right)\right|^{s} \\
& \quad \leq C_{s} \sum_{k=1}^{n} E\left|f_{n k}\left(X_{k}\right)\right|^{s}+g(n, s)\left(\sum_{k=1}^{n} E\left(f_{n k}\left(X_{k}\right)\right)^{2}\right)^{s / 2}, \quad \forall n \geq 1, s \geq 2,
\end{aligned}
$$

and

$$
\begin{aligned}
& E \max _{1 \leq m \leq n}\left|\sum_{k=1}^{m}\left(f_{n k}\left(X_{k}\right)-E f_{n k}\left(X_{k}\right)\right)\right|^{s} \\
& \quad \leq C_{s} \sum_{k=1}^{n} E\left|f_{n k}\left(X_{k}\right)\right|^{s}+g(n, s)\left(\sum_{k=1}^{n} E\left(f_{n k}\left(X_{k}\right)\right)^{2}\right)^{s / 2}, \quad \forall n \geq 1, s \geq 2,
\end{aligned}
$$

where $g(x, y)$ is a positive function.

A sequence of random variables $\left\{X_{n}, n \geq 1\right\}$ is said to be widely upper orthant dependent (WUOD) if, for each $n \geq 1$, there exists a positive number $g_{U}(n)$ such that, for all real numbers $x_{i}, 1 \leq i \leq n$,

$$
P\left(X_{1}>x_{1}, \ldots, X_{n}>x_{n}\right) \leq g_{U}(n) \prod_{i=1}^{n} P\left(X_{i}>x_{i}\right)
$$

It is said to be widely lower orthant dependent (WLOD) if, for each $n \geq 1$, there exists a positive number $g_{L}(n)$ such that, for all real numbers $x_{i}, 1 \leq i \leq n$,

$$
P\left(X_{1} \leq x_{1}, \ldots, X_{n} \leq x_{n}\right) \leq g_{L}(n) \prod_{i=1}^{n} P\left(X_{i} \leq x_{i}\right)
$$

and it is said to be widely orthant dependent (WOD) if it is both WUOD and WLOD. The sequences $\left\{g_{U}(n), n \geq 1\right\}$ and $\left\{g_{L}(n), n \geq 1\right\}$ are called dominating coefficients (see Wang et al. [10]). If for all $n \geq 1, g_{U}(n)=g_{L}(n)=M$ for some positive constant $M$, then $\left\{X_{n}, n \geq 1\right\}$ is said to be extended negatively dependent (END). In particular, if $M=1$, then $\left\{X_{n}, n \geq 1\right\}$ is said to be negatively orthant dependent (NOD) or negatively dependent. Since the class of WOD random variables contains END random variables and NOD random variables as special cases, it is interesting to study the limiting behavior of WOD random variables.

If $\left\{X_{n}, n \geq 1\right\}$ is a sequence of WOD random variables with the dominating coefficients $g_{L}(n)$ and $g_{U}(n)$ for $n \geq 1$, then (1.5) holds with $g(n, s)=D_{s}\left(g_{L}(n)+g_{U}(n)\right)$, where $D_{s}$ is a positive constant depending only on $s$ (see Chen and Sung [4]).

In this paper, we extend the Cuzick-Bai-Cheng result to random variables satisfying (1.5) or (1.6) with a suitable condition on $g(x, y)$. In particular, under (1.6), we obtain the 
following strong law which is slightly stronger than (1.1):

$$
n^{-1 / p} \max _{1 \leq m \leq n}\left|\sum_{k=1}^{m} a_{n k} X_{k}\right| \rightarrow 0 \quad \text { a.s. }
$$

We also extend the Cuzick-Bai-Cheng result to WOD random variables.

The rest of this paper is organized as follows. In Sect. 2, we present the main results. The proofs of the results in Sect. 2 are given in Sect. 3.

Throughout this paper, the symbol $C$ denotes a positive constant which is not necessarily the same one in each appearance. For an event $A, I(A)$ denotes the indicator function of the event $A$. For a real number $x, x^{+}=\max \{x, 0\}$ and $x^{-}=\max \{-x, 0\}$.

\section{Main results}

We first present strong laws for weighted sums of random variables satisfying (1.5) or (1.6). It is necessary to limit the growth of $g(x, y)$ in (1.5) and (1.6). From now on, we always assume that there exists a constant $\tau \in[0, \infty)$ such that $g(x, y)=O\left(x^{\tau}\right)$ as $x \rightarrow \infty$ for any fixed $y>0$.

Theorem 2.1 Let $1 \leq p<2$ and $\alpha, \beta>0$ with $1 / p=1 / \alpha+1 / \beta$. Let $\left\{X_{n}, n \geq 1\right\}$ be a sequence of mean zero random variables satisfying (1.5) for any nondecreasing functions $\left\{f_{n k}(x)\right\}$ and stochastically dominated by a random variable $X$ satisfying $E|X|^{\beta}<\infty$. Let $\left\{a_{n k}, 1 \leq k \leq\right.$ $n, n \geq 1\}$ be an array of constants satisfying (1.2). Then (1.1) holds.

If condition (1.5) is replaced by a stronger condition (1.6), then we have a stronger result.

Theorem 2.2 Let $1 \leq p<2$ and $\alpha, \beta>0$ with $1 / p=1 / \alpha+1 / \beta$. Let $\left\{X_{n}, n \geq 1\right\}$ be a sequence of mean zero random variables satisfying (1.6) for any nondecreasing functions $\left\{f_{n k}(x)\right\}$ and stochastically dominated by a random variable $X$ satisfying $E|X|^{\beta}<\infty$. Let $\left\{a_{n k}, 1 \leq k \leq\right.$ $n, n \geq 1\}$ be an array of constants satisfying (1.2). Then (1.7) holds.

In Theorems 2.1 and 2.2, we have considered only the case $1 \leq p<2$. If $0<p<1$, then (1.1) and (1.7) hold without any conditions imposed on the joint distributions of the random variables.

Theorem 2.3 Let $0<p<1$ and $\alpha, \beta>0$ with $1 / p=1 / \alpha+1 / \beta$. Let $\left\{X_{n}, n \geq 1\right\}$ be a sequence of random variables which are stochastically dominated by a random variable $X$ satisfying $E|X|^{\beta}<\infty$. Let $\left\{a_{n k}, 1 \leq k \leq n, n \geq 1\right\}$ be an array of constants satisfying (1.2). Then

$$
n^{-1 / p} \sum_{k=1}^{n}\left|a_{n k}\right|\left|X_{k}\right| \rightarrow 0 \quad \text { a.s. }
$$

and hence (1.1) and (1.7) hold.

Remark 2.1 Wu et al. [13] proved Theorems 2.1 and 2.3 under stronger conditions. They proved Theorem 2.3 under an additional condition (1.4). When $p=1$, they proved Theorem 2.1 under a stronger condition (1.4) than (1.5). When $1<p<2$, they proved Theorem 2.1 under a special condition (1.3) and a stronger moment condition $E|X|^{u}<\infty$ for some $u>\beta$. 
Remark 2.2 As mentioned in Introduction, (1.3) holds for negatively orthant dependent random variables. Hence, Theorem 2.1 holds for negatively orthant dependent random variables. On the other hand, $\mathrm{Wu}$ [12] already proved Theorem 2.1 for such random variables. Wu [12] also proved Theorem 2.3 under the stronger condition that $\left\{X_{n}, n \geq 1\right\}$ are negatively orthant dependent random variables.

Remark 2.3 As mentioned in Introduction, (1.4) holds for $\varphi$-mixing random variables satisfying a mixing rate condition $\sum_{n=1}^{\infty} \varphi^{1 / 2}(n)<\infty$. Hence, Theorem 2.2 holds for $\varphi$-mixing random variables with $\sum_{n=1}^{\infty} \varphi^{1 / 2}(n)<\infty$. On the other hand, Huang et al. [6] already proved Theorem 2.2 for such random variables. They also proved Theorem 2.3 under the stronger condition that $\left\{X_{n}, n \geq 1\right\}$ are $\varphi$-mixing random variables with $\sum_{n=1}^{\infty} \varphi^{1 / 2}(n)<\infty$.

We next present a strong law for weighted sums of WOD random variables.

Theorem 2.4 Let $1 \leq p<2$ and $\alpha, \beta>0$ with $1 / p=1 / \alpha+1 / \beta$. Let $\left\{X, X_{n}, n \geq 1\right\}$ be a sequence of identically distributed WOD random variables with dominating coefficients $g_{L}(n)$ and $g_{U}(n)$ for $n \geq 1$. Suppose that there exist a positive function $g(x)$ for $x \geq 0$ and a nonnegative constant $0 \leq \tau<\infty$ such that $g(x)=O\left(x^{\tau}\right)$ and $\max \left\{g_{L}(n), g_{U}(n)\right\} \leq g(n)$ for $n \geq 1$. Let $\left\{a_{n k}, 1 \leq k \leq n, n \geq 1\right\}$ be an array of constants satisfying (1.2). Assume that $E X=0$ and $E|X|^{\beta}<\infty$. Then (1.1) holds.

Corollary 2.1 Let $1 \leq p<2$. Let $\left\{X, X_{n}, n \geq 1\right\}$ be a sequence of identically distributed WOD random variables with dominating coefficients $g_{L}(n)$ and $g_{U}(n)$ for $n \geq 1$. Suppose that there exist a positive function $g(x)$ for $x \geq 0$ and a nonnegative constant $0 \leq \tau<\infty$ such that $g(x)=O\left(x^{\tau}\right)$ and $\max \left\{g_{L}(n), g_{U}(n)\right\} \leq g(n)$ for $n \geq 1$. Assume that $E X=0$ and $E|X|^{\beta}<\infty$ for some $\beta>p$. Then

$$
n^{-1 / p} \sum_{k=1}^{n} X_{k} \rightarrow 0 \quad \text { a.s. }
$$

\section{Proofs}

In this section, we present the proofs of the results in Sect. 2.

Proof of Theorem 2.1 We can rewrite (1.1) as

$$
n^{-1 / p} \sum_{k=1}^{n} a_{n k} X_{k}=n^{-1 / p} \sum_{k=1}^{n} a_{n k}^{+} X_{k}-n^{-1 / p} \sum_{k=1}^{n} a_{n k}^{-} X_{k} .
$$

Hence, we may assume that $a_{n k} \geq 0$ for all $1 \leq k \leq n$ and $n \geq 1$. For $1 \leq k \leq n$ and $n \geq 1$, let

$$
\begin{aligned}
& Y_{n k}=X_{k} I\left(\left|X_{k}\right| \leq n^{1 / \beta}\right)+n^{1 / \beta} I\left(X_{k}>n^{1 / \beta}\right)-n^{1 / \beta} I\left(X_{k}<-n^{1 / \beta}\right), \\
& Z_{n k}=X_{k}-Y_{n k} .
\end{aligned}
$$

Then

$$
n^{-1 / p} \sum_{k=1}^{n} a_{n k} X_{k}=n^{-1 / p} \sum_{k=1}^{n} a_{n k} Z_{n k}+n^{-1 / p} \sum_{k=1}^{n} a_{n k} E Y_{n k}+n^{-1 / p} \sum_{k=1}^{n} a_{n k}\left(Y_{n k}-E Y_{n k}\right)
$$




$$
:=I_{1}+I_{2}+I_{3}
$$

We first note that $\left|Z_{n k}\right| \leq\left|X_{k}\right| I\left(\left|X_{k}\right|>n^{1 / \beta}\right)$. Since $\sum_{n=1}^{\infty} P\left(\left|X_{n}\right|>n^{1 / \beta}\right) \leq D \sum_{n=1}^{\infty} P(|X|>$ $\left.n^{1 / \beta}\right) \leq D E|X|^{\beta}<\infty$, we have by the Borel-Cantelli lemma that

$$
\begin{aligned}
\left|I_{1}\right| & \leq n^{-1 / p} \max _{1 \leq k \leq n}\left|a_{n k}\right| \sum_{k=1}^{n}\left|Z_{n k}\right| \\
& \leq C n^{-1 / p} n^{1 / \alpha} \sum_{k=1}^{n}\left|X_{k}\right| I\left(\left|X_{k}\right|>n^{1 / \beta}\right) \\
& \leq C n^{-1 / \beta} \sum_{k=1}^{\infty}\left|X_{k}\right| I\left(\left|X_{k}\right|>k^{1 / \beta}\right) \\
& \rightarrow 0 \quad \text { a.s. }
\end{aligned}
$$

On account of $E X_{n}=0$ for all $n \geq 1$, we obtain that

$$
\begin{aligned}
\left|I_{2}\right| & =n^{-1 / p}\left|\sum_{k=1}^{n} a_{n k} E Z_{n k}\right| \\
& \leq n^{-1 / p} \sum_{k=1}^{n}\left|a_{n k}\right| E\left|Z_{n k}\right| \\
& \leq D n^{-1 / p} E|X| I\left(|X|>n^{1 / \beta}\right) \sum_{k=1}^{n}\left|a_{n k}\right| \\
& \leq C n^{1-1 / p} E|X| I\left(|X|>n^{1 / \beta}\right) \\
& \leq C n^{-1 / \alpha} E|X|^{\beta} I\left(|X|>n^{1 / \beta}\right) \\
& \rightarrow 0 .
\end{aligned}
$$

Finally, we prove that $I_{3} \rightarrow 0$ a.s. By the Borel-Cantelli lemma, it suffices to show that

$$
\sum_{n=1}^{\infty} P\left(\left|I_{3}\right|>\varepsilon\right)<\infty, \quad \forall \varepsilon>0
$$

We prove (3.1) by using (1.5). To do this, let $f_{n k}(x)=a_{n k} h_{n}(x)$, where $h_{n}(x)=x I(|x| \leq$ $\left.n^{1 / \beta}\right)+n^{1 / \beta} I\left(x>n^{1 / \beta}\right)-n^{1 / \beta} I\left(x<-n^{1 / \beta}\right)$. Then $f_{n k}(x), 1 \leq k \leq n, n \geq 1$, are nondecreasing functions, and $f_{n k}\left(X_{k}\right)=a_{n k} Y_{n k}$. Taking $s>(\tau+1) \cdot \max \{\alpha, \beta, 2 p /(2-p)\}$, we have by the Markov inequality and (1.5) that

$$
\begin{aligned}
& \sum_{n=1}^{\infty} P\left(\left|I_{3}\right|>\varepsilon\right) \\
& \quad \leq \varepsilon^{-s} \sum_{n=1}^{\infty} n^{-s / p} E\left|\sum_{k=1}^{n} a_{n k}\left(Y_{n k}-E Y_{n k}\right)\right|^{s} \\
& \quad \leq C \sum_{n=1}^{\infty} n^{-s / p}\left\{\sum_{k=1}^{n} a_{n k}^{s} E\left|Y_{n k}\right|^{s}+g(n, s)\left(\sum_{k=1}^{n} a_{n k}^{2} E Y_{n k}^{2}\right)^{s / 2}\right\}
\end{aligned}
$$




$$
:=C\left\{I_{4}+I_{5}\right\}
$$

Since $s>\alpha$, we have that $\sum_{k=1}^{n} a_{n k}^{s}=O\left(n^{s / \alpha}\right)$. By the stochastic domination condition, we also have that $E\left|Y_{n k}\right|^{s}=E\left|X_{k}\right|^{s} I\left(\left|X_{k}\right| \leq n^{1 / \beta}\right)+n^{s / \beta} P\left(\left|X_{k}\right|>n^{1 / \beta}\right) \leq D E|X|^{s} I\left(|X| \leq n^{1 / \beta}\right)+$ $2 D n^{s / \beta} P\left(|X|>n^{1 / \beta}\right)$. It follows that

$$
\begin{aligned}
I_{4} & \leq C \sum_{n=1}^{\infty} n^{-s / p} \sum_{k=1}^{n} a_{n k}^{s}\left\{D E|X|^{s} I\left(|X| \leq n^{1 / \beta}\right)+2 D n^{s / \beta} P\left(|X|>n^{1 / \beta}\right)\right\} \\
& \leq C \sum_{n=1}^{\infty} n^{-s / p} n^{s / \alpha}\left\{D E|X|^{s} I\left(|X| \leq n^{1 / \beta}\right)+2 D n^{s / \beta} P\left(|X|>n^{1 / \beta}\right)\right\} \\
& =C D \sum_{n=1}^{\infty} n^{-s / \beta} E|X|^{s} I\left(|X| \leq n^{1 / \beta}\right)+2 C D \sum_{n=1}^{\infty} P\left(|X|>n^{1 / \beta}\right) \\
& \leq C E|X|^{\beta}<\infty .
\end{aligned}
$$

We show that $I_{5}<\infty$ with two cases.

When $\beta<2$, we have that $\alpha>2$ and $E\left|Y_{n k}\right|^{2}=E\left|X_{k}\right|^{2} I\left(\left|X_{k}\right| \leq n^{1 / \beta}\right)+n^{2 / \beta} P\left(\left|X_{k}\right|>n^{1 / \beta}\right) \leq$ $D E|X|^{2} I\left(|X| \leq n^{1 / \beta}\right)+2 D n^{2 / \beta} P\left(|X|>n^{1 / \beta}\right) \leq D n^{(2-\beta) / \beta} E|X|^{\beta} I\left(|X| \leq n^{1 / \beta}\right)+$ $2 D n^{(2-\beta) / \beta} E|X|^{\beta} I\left(|X|>n^{1 / \beta}\right) \leq 2 D n^{(2-\beta) / \beta} E|X|^{\beta}$. It follows that

$$
\begin{aligned}
I_{5} & \leq \sum_{n=1}^{\infty} n^{-s / p} g(n, s)\left(\sum_{k=1}^{n} a_{n k}^{2} 2 D n^{(2-\beta) / \beta} E|X|^{\beta}\right)^{s / 2} \\
& \leq C \sum_{n=1}^{\infty} n^{-s / p+\tau} n^{(2-\beta) s /(2 \beta)}\left(\sum_{k=1}^{n} a_{n k}^{2}\right)^{s / 2} \\
& \leq C \sum_{n=1}^{\infty} n^{-s / p+\tau} n^{(2-\beta) s /(2 \beta)} n^{s / 2} \\
& =C \sum_{n=1}^{\infty} n^{-s / \alpha+\tau} \\
& <\infty
\end{aligned}
$$

since $s>\alpha(\tau+1)$.

When $\beta \geq 2$, we have that $E\left|Y_{n k}\right|^{2} \leq E\left|X_{k}\right|^{2} \leq D E|X|^{2}<\infty$. It follows that

$$
\begin{aligned}
I_{5} & \leq C \sum_{n=1}^{\infty} n^{-s / p} g(n, s)\left(\sum_{k=1}^{n} a_{n k}^{2}\right)^{s / 2} \\
& \leq \begin{cases}C \sum_{n=1}^{\infty} n^{-s / p+\tau} n^{s / \alpha} & \text { if } \alpha \leq 2, \\
C \sum_{n=1}^{\infty} n^{-s / p+\tau} n^{s / 2} & \text { if } \alpha>2,\end{cases} \\
& <\infty,
\end{aligned}
$$

since $s>\beta(\tau+1)$ and $s>2 p(\tau+1) /(2-p)$. The proof is completed.

Proof of Theorem 2.2 The proof is similar to that of Theorem 2.1 and is omitted. 
Proof of Theorem 2.3 When the random variables $\left\{X_{n}, n \geq 1\right\}$ are identically distributed, the proof is proved by Chen and Gan [3]. For the non-identically distributed case, the proof is the same as that of Chen and Gan [3], and thus it is omitted.

Proof of Theorem 2.4 By Lemma 2.6 in Chen and Sung [4], all conditions of Theorem 2.1 are satisfied. Hence the result follows directly from Theorem 2.1.

Proof of Corollary 2.1 Set $a_{n k}=1$ for all $n \geq 1$ and $1 \leq k \leq n$. Then (1.2) holds for $\alpha=$ $p \beta /(\beta-p)$, and hence the result follows directly from Theorem 2.4.

Acknowledgements

Not applicable.

\section{Funding}

The research of Yanchun Yi is supported by Hunan Provincial Natural Science Foundation of China (2018JJ4024), and the Key Projects of Hunan Provincial Education Department (18A3400). The research of Pingyan Chen is supported by the National Natural Science Foundation of China (No. 71471075). The research of Soo Hak Sung is supported by the Basic Science Research Program through the National Research Foundation of Korea (NRF) funded by the Ministry of Education (2017R1D1A1B03029898)

\section{Availability of data and materials}

Not applicable.

\section{Competing interests}

The authors declare that they have no competing interests.

Authors' contributions

All authors read and approved the manuscript.

\section{Author details}

${ }^{1}$ College of Math. and Statist., Hengyang Normal University, Hengyang, P.R. China. ${ }^{2}$ Department of Mathematics, Jinan University, Guangzhou, P.R. China. ${ }^{3}$ Department of Applied Mathematics, Pai Chai University, Daejeon, South Korea.

\section{Publisher's Note}

Springer Nature remains neutral with regard to jurisdictional claims in published maps and institutional affiliations.

Received: 28 October 2019 Accepted: 11 February 2020 Published online: 19 February 2020

\section{References}

1. Asadian, N., Fakoor, V., Bozorgnia, A.: Rosenthal's type inequalities for negatively orthant dependent random variables. J. Iran. Stat. Soc. 5, 69-75 (2006)

2. Bai, Z.D., Cheng, P.E.: Marcinkiewicz strong laws for linear statistics. Stat. Probab. Lett. 46, 105-112 (2000)

3. Chen, P., Gan, S.: Limiting behavior of weighted sums of i.i.d. random variables. Stat. Probab. Lett. 77, 1589-1599 (2007)

4. Chen, P., Sung, S.H.: A Spitzer-type law of large numbers for widely orthant dependent random variables. Stat. Probab. Lett. (2019). https://doi.org/10.1016/j.spl.2019.06.020

5. Cuzick, J.: A strong law for weighted sums of i.i.d. random variables. J. Theor. Probab. 8, 625-641 (1995)

6. Huang, H., Wang, D., Peng, J.: On the strong law of large numbers for weighted sums of $\varphi$-mixing random variables. J. Math. Inequal. 8, 465-473 (2014)

7. Rosenthal, H.P.: On the subspaces of $L^{P}(p>2)$ spanned by sequences of independent random variables. Isr. J. Math. 8, 273-303 (1970)

8. Shao, Q.M.: A comparison theorem on moment inequalities between negatively associated and independent random variables. J. Theor. Probab. 13, 343-356 (2000)

9. Utev, S., Peligrad, M.: Maximal inequalities and an invariance principle for a class of weakly dependent random variables. J. Theor. Probab. 16, 101-115 (2003)

10. Wang, K., Wang, Y., Gao, Q.: Uniform asymptotics for the finite-time ruin probability of a dependent risk model with a constant interest rate. Methodol. Comput. Appl. Probab. 15, 109-124 (2013)

11. Wang, X., Hu, S., Yang, W., Shen, Y.: On complete convergence for weighted sums of $\varphi$-mixing random variables. J. Inequal. Appl. 2010, Article ID 372390 (2010)

12. Wu, Q: A strong limit theorem for weighted sums of sequences of negatively dependent random variables. J. Inequal. Appl. 2010, Article ID 383805 (2010)

13. Wu, Y., Wang, X., Hu, S., Yang, L.: Weighted version of strong law of large numbers for a class of random variables and its applications. Test 27, 379-406 (2018) 